\title{
Influence of the drug
}

exposure definition on the

assessment of the

\section{antipsychotic metabolic} impact in patients initially

\section{treated with mood-stabilizers}

Marie Tournier, ${ }^{1,2,3}$ Bernard Bégaud, ${ }^{1,2,4}$ Audrey Cougnard, ${ }^{2}$

Guy-Robert Auleley, ${ }^{5,6}$ Jean Deligne, ${ }^{5}$ Claudine Blum-Boisgard, ${ }^{5}$

Anne C. M. Thiébaut ${ }^{2,7} \&$ Hélène Verdoux ${ }^{1,2,3}$

'Université de Bordeaux, U657, ${ }^{2}$ INSERM, U657, ${ }^{3}$ Centre Hospitalier Charles Perrens, ${ }^{4}$ Centre Hospitalier Universitaire de Bordeaux, F-33000 Bordeaux, ${ }^{5}$ Caisse Nationale du Régime Social des Indépendants, National Medical Department, Paris, ${ }^{6}$ National Cancer Institute, Clinical Research Department, Boulogne-Billancourt and ${ }^{7}$ Institut Pasteur, Unité de Pharmaco-Epidémiologie et Maladies Infectieuses (PhEMI), Paris, France

\section{WHAT IS ALREADY KNOWN ABOUT} THIS SUBJECT

- Metabolic disturbances represent a well-known side effect of second generation antipsychotics. However, studies comparing second generation antipsychotic drugs (SGAPs) and first generation antipsychotic drugs (FGAPs) through administrative databases have shown contrasting findings, which may be attributable to methodological differences.

\section{WHAT THIS PAPER ADDS}

- The definition of antipsychotic exposure impacts on the association between antipsychotics and metabolic risk in studies carried out through administrative databases.

- Considering cumulative exposure to antipsychotics or including patients exposed to an antipsychotic drug for months or years is likely to over-represent patients who tolerate the drug well with a depletion of susceptible effects. - Antipsychotic drug exposure is a time-varying determinant and episodes of no use, past use and current use should be distinguished over the study period to avoid any misclassification bias that might lead to misleading findings.

\section{METHODS}

\section{AIMS}

To assess the influence of three definitions of antipsychotic exposure on the comparison between first generation (FGAP) and second generation (SGAP) antipsychotic drugs and 'conventional' mood stabilizers towards the risk of metabolic events using (i) a dichotomous measure (exposed/non-exposed over the follow-up), (ii) a categorical measure taking into account the chronology of exposure at the time of the metabolic event (current, recent and no use) and (iii) a continuous measure (cumulative duration).

A historical fixed cohort was identified from the 2004-2006 claims database of the French health insurance programme for self-employed workers, including 3172 patients aged 18 years and over who used conventional mood stabilizers over a 3 month period. A metabolic event was defined as an incident dispensing of an anti-diabetic or lipid-lowering drug.

\section{RESULTS}

A metabolic event occurred in 367 patients (11.6\%). At least one FGAP had been prescribed in $29 \%$ of patients who did not develop a metabolic event and in $22 \%$ of patients who developed a metabolic event. In addition, at least one SGAP had been prescribed in $12 \%$ of patients who did not develop a metabolic event and in $7 \%$ of patients who developed a metabolic event. Compared with conventional mood stabilizers, the risk of a metabolic event was negatively associated with exposure to SGAPs over the follow-up period (HR $0.53,95 \% \mathrm{Cl} 0.34,0.82, P=0.004$ ), positively associated with recent, but not current, exposure to SGAPs (HR 2.1, 95\% $\mathrm{Cl} 1.2,3.7, P=$ $0.006)$ and not associated with cumulative duration of SGAPs (HR 1.001, $95 \% \mathrm{Cl} 0.999$, $1.003, P=0.20$ )

\section{CONCLUSIONS}

The definition of exposure to antipsychotics in epidemiological studies exploring their metabolic impact is of paramount importance in understanding this association. Different definitions can lead to opposite and seemingly nonsensical results. Not taking into account past exposure, in order to minimize the depletion of susceptible effects, may lead to absurd results. 


\section{Introduction}

While second generation antipsychotics (SGAPs) may lead to advantages over first generation antipsychotics (FGAPs) regarding extrapyramidal side effects [1], they may be more likely to cause or exacerbate several metabolic disorders such as weight gain, dyslipidaemia, diabetes, metabolic syndrome and ultimately cardiovascular disease [2-4]. Since patients included and patterns of use in randomized control trials exploring the efficacy of antipsychotics were unlikely to be representative of real-life practice, this issue may be explored using data collected in naturalistic settings. However, studies comparing SGAPs and FGAPs through administrative databases have shown contrasting findings, probably attributable to methodological differences. In particular, the definition of antipsychotic exposure might impact on the association between antipsychotics and metabolic risk. Most studies concluding no difference between FGAPs and SGAPs considered the duration of antipsychotic exposure [5, 6]. A study using antipsychotic cumulative time of exposure found a hardly significant and weak association between SGAP use and diabetes, compared with FGAPs [7]. On the contrary, in studies finding that SGAP use was associated with an increased risk of diabetes compared with FGAP use, the exposure to antipsychotic was defined as a dichotomous variable $[8,9]$. In studies conducted through the UK General Practice Research Database, olanzapine use was found to be significantly associated with diabetes compared with FGAP use when antipsychotic exposure was defined as at least one prescription within the 3 preceding months, but not when the incidence rate of diabetes was expressed in person-years of exposure $[1,10]$.

Furthermore, other methodological options might influence the findings. For example, studies including patients exposed to a drug treatment for months or years are likely to over-represent patients who tolerate the drug well $[8,9,11]$. Recent discontinuation of a drug treatment may be promoted by the occurrence of a side effect that persists for a while after treatment discontinuation.

Although SGAPs are increasingly used in the treatment of bipolar disorders instead of conventional mood stabilizers (lithium or anticonvulsants) [12-14], few studies have been carried out to compare antipsychotics with mood stabilizers in patients with bipolar disorder. A nested casecontrol study conducted in patients with bipolar disorder in the Medicaid managed care programme showed that the risk of diabetes was higher among those taking risperidone, olanzapine and quetiapine, compared with those receiving FGAPs [9]. In another case-control study conducted using a US medical claims database, the risk of diabetes was higher among patients taking clozapine, risperidone, olanzapine and quetiapine, compared with those receiving FGAPs [8]. In these studies, antipsychotic exposure was defined as at least 3 months of treatment or at least three prescriptions during the study period.
Studies restricted to patients with bipolar disorder allow comparison of the risk of adverse metabolic outcome in antipsychotic users with that of persons treated with other treatments. Indeed, such a comparison is more complex in studies carried out in persons with non-affective psychosis, as periods with no antipsychotic treatment are usually due to poor treatment adherence, which itself may be associated with life-style characteristics increasing the risk of metabolic disturbances. Conventional mood stabilizers are reported to induce weight gain but this effect appears less frequent and of less magnitude than what is observed with SGAPs $[11,15]$.

The present study assessed the influence of using three definitions of antipsychotic exposure on the comparison between antipsychotic drugs (FGAPs and SGAPs) and 'conventional' mood stabilizers towards the risk of metabolic events.

\section{Methods}

\section{Design}

A historical fixed cohort study was carried out in patients aged 18 years and over who were dispensed conventional mood stabilizers (lithium or anticonvulsants) at least over a 3 month period [16]. The association between incident dispensing of an antipsychotic drug and an incident metabolic event was assessed over a 2 year follow-up period using three different definitions of antipsychotic exposure.

\section{Setting and study population}

Data were anonymously extracted from the 2004-2006 database of the Régime Social des Travailleurs Indépendants (RSI), the French national health care insurance programme for self-employed workers (artisans, tradespeople, physicians, lawyers) and their dependents. In 2006, 4 million persons were covered by the RSI. The RSI database includes information on all community claims for reimbursement of prescriptions dispensed for all drugs on the market classified using the WHO Anatomical Therapeutic Chemical (ATC) classification system [17].

As no information on diagnoses was directly available in the RSI database, exposure to a mood stabilizer drug treatment included those with a marketing authorization restricted to bipolar disorder (i.e. lithium, valpromide and/or sodium divalproate) in order not to include patients treated with anticonvulsants for epilepsy-related disorders or other indications. For example carbamazepine was excluded since it is marketed both for mania and epilepsy. No other anticonvulsant had marketing authorization for mood disorders in France at the time of the survey. To define the 3 month inclusion period of continuous dispensing between January 12004 and December 31 2004, we considered that discontinuation of mood stabilizer treatment occurred in the event of non-renewal of dispensing during the 28 days following the predicted end of 
the previous dispensing, since dispensings are issued for a maximum of 28 days. The index date was defined as the end of this 3 month inclusion period.

To focus on incident antipsychotic dispensing and incident metabolic events, patients with a dispensing of antipsychotic, anti-diabetic or lipid-lowering drugs over this 3 month period were excluded. Since sedative antipsychotic drugs (cyamemazine, levomepromazine) are commonly used as anxiolytic or hypnotic drugs in patients treated with mood stabilizers, exclusion of subjects with a prevalent dispensing may markedly reduce the sample size. Hence, we performed two series of analyses, the first one keeping and the second excluding subjects treated with these drugs over the 3 month inclusion period, in order to assess whether the findings were altered.

\section{Definition of drug exposure}

The main independent variable was exposure to antipsychotic drug treatment. Three different definitions of antipsychotic exposure were used. First, we considered a dichotomous definition of exposure: at least one dispensing of an antipsychotic drug vs. no dispensing over the study period. Second, we considered a continuous measure of antipsychotic exposure defined as cumulative time of exposure (in days). In the event of discontinuation of antipsychotic treatment, the length of exposure went back to square one after 6 months of interruption since an incident metabolic event occurring more than 6 months after discontinuation of the treatment was unlikely to be attributable to the treatment. Third, we considered a categorical definition taking into account the pattern of exposure at the time of the metabolic event: (i) current use, (ii) recent use (no current use but use over the past 6 months) and (iii) no use (no dispensing over the last 6 months).

Antipsychotics marketed in France at the time of the study were classified as FGAPs (i.e. cyamemazine, chlorpromazine, droperidol, flupentixol, fluphonazine, haloperidol, levopromazine, loxapine, penfluridol, periciazine, perphenazine, pimozide, pipamperon, pipotiazine, sulpiride, sultopride and zuclopentixol) and SGAPs (i.e. amisulpride, aripiprazole, clozapine, olanzapine and risperidone).

The category of mood stabilizers was more broadly defined over the follow-up period than in the inclusion period and included those approved for bipolar disorder (i.e. lithium, valpromide, divalproate sodium, carbamazepine) as well as those used empirically in bipolar disorder without formal approval (i.e. lamotrigine and oxcarbazepine). We considered that incident dispensings of anticonvulsants approved both for epilepsy and bipolar disorder occurring after the 3 month inclusion period were for mood stabilizing purposes.

\section{Covariates}

We considered as covariates age, gender and nonpsychotropic drugs known to induce metabolic events: diuretics, $\alpha$-adrenoceptor blockers, $\beta$-adrenoceptor blockers, corticosteroids, phenytoin, thyroid hormones, amiodarone, androgens, oral contraceptives containing norgesterol or androgen-oestrogen combination and second generation oral progestogens [18].

\section{Occurrence of a metabolic event}

The occurrence of a metabolic event over the follow-up period was conservatively defined as at least one incident dispensing of an anti-diabetic drug as a marker of diabetes or at least one incident dispensing of a lipid-lowering drug as a marker of hyperlipidaemia (hypercholesterolaemia or hypertriglyceridaemia). In order to take into account that it is recommended to treat incident metabolic disturbances using only lifestyle and nutritional interventions during 6 months before prescribing a drug treatment $[19,20]$, we considered that a metabolic event may be attributed to the drug treatment during 6 months after treatment discontinuation and explored the occurrence of a metabolic event in patients with 'current' or 'recent' (discontinued for less than 6 months) antipsychotic exposure.

\section{Statistical analyses}

The three different definitions of antipsychotic exposure were used consecutively. We investigated the metabolic impact of exposure to antipsychotic drug treatments compared with conventional mood stabilizers using Cox proportional hazards regression models with person-days of follow-up as the primary time variable [21]. Person-days of follow-up were calculated from the end of the 3 month period to the dispensing date of an anti-diabetic drug or a lipid-lowering drug, or to the end of the follow-up period. Hazard ratios (HRs) and 95\% confidence intervals (Cls) were estimated using the Efron approximation to handle events occurring at the same time in different patients (ties) [22]. Follow-up ended at the date of the first of the following events: (i) occurrence of a metabolic event, (ii) change of health insurance programme, (iii) death or (iv) end of the study period, i.e. date of last medication dispensing whatever the medication or date of last medical service in the claim database. For each definition of drug exposure, to take co-dispensing into account, all categories of antipsychotic treatments were simultaneously entered in the multivariate models. We defined at least one dispensing of a mood stabilizer over the follow-up as our reference category for the dichotomous variable and current use of mood stabilizers as our reference category for the categorical and continuous variables. All the analyses were a priori adjusted for age and gender.

For each definition of antipsychotic exposure, three outcomes were investigated: (i) any metabolic event (new dispensing of an anti-diabetic drug or of a lipid-lowering drug), (ii) a new dispensing of an anti-diabetic drug or (iii) a new dispensing of a lipid-lowering drug. Then, in order to select only incident exposure to all antipsychotics, the same models were performed excluding patients exposed 
to sedative FGAPs (cyamemazine and levomepromazine) over the 3 month inclusion period. Finally, the impact of $a$ priori defined covariates was explored by entering nonpsychotropic drugs likely to induce metabolic events one at a time into the models. All statistical analyses were conducted using STATA 9 software [23].

\section{Results}

\section{Description of study population}

The characteristics of the 3170 subjects included in the study population are detailed in Table 1. An incident meta- bolic event was observed in 367 patients (11.6\%), a new anti-diabetic dispensing being much less frequent $(n=62$; $2 \%)$ than a new lipid-lowering drug dispensing $(n=328$; 10.3\%). Patients with an incident metabolic event were less often exposed to any kind of drugs likely to induce metabolic events over the study period than patients without a metabolic event (Table 1).

\section{First method: exposure to antipsychotics over the follow-up}

When exposure to antipsychotic drugs was defined as at least one dispensing over the follow-up period (Table 2), patients with an incident metabolic event and patients

\section{Table 1}

Exposure to drugs according to occurrence of an incident metabolic event

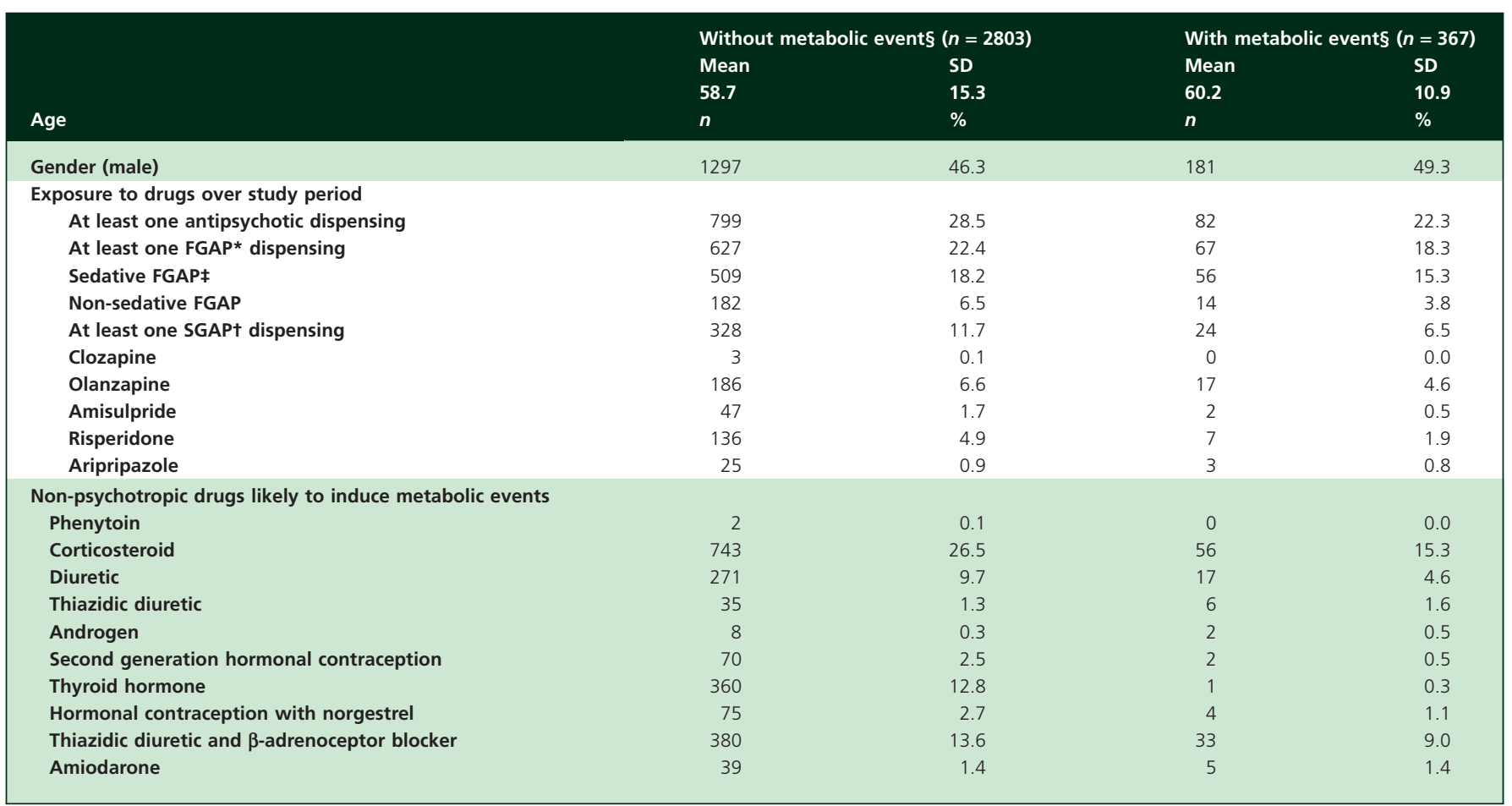

*First generation antipsychotic; †Second generation antipsychotic; ¥Levopromazine, cyamemazine; §Dispensing of an anti-diabetic or lipid-lowering drugs.

\section{Table 2}

Association between at least one dispensing of antipsychotic drug over the follow-up period* and occurrence of incident metabolic event +

\begin{tabular}{|c|c|c|c|c|c|c|c|c|c|}
\hline \multirow[b]{2}{*}{ In total sample } & \multicolumn{3}{|c|}{ Incident anti-diabetic dispensing } & \multicolumn{3}{|c|}{ Incident lipid-lowering drug dispensing } & \multicolumn{3}{|c|}{ Any incident metabolic event ${ }^{\dagger}$} \\
\hline & HR & $95 \%$ CI§ & $P$ & HR & $95 \% \mathrm{Cl}$ & $P$ & HR & $95 \% \mathrm{CI}$ & $P$ \\
\hline Exposure to SGAPף & 0.32 & $0.08,1.34$ & 0.12 & 0.59 & $0.38,0.93$ & 0.024 & 0.53 & $0.34,0.82$ & 0.004 \\
\hline Exposure to FGAP** & 0.97 & $0.48,1.96$ & 0.93 & 0.83 & $0.61,1.12$ & 0.22 & 1.86 & $0.65,1.13$ & 0.27 \\
\hline Exposure to SGAPף & 0.42 & $0.10,1.78$ & 0.24 & 0.61 & $0.38,1.00$ & 0.05 & 0.56 & $0.35,0.89$ & 0.02 \\
\hline Exposure to FGAP** & 0.70 & $0.25,1.99$ & 0.51 & 0.74 & $0.50,1.10$ & 0.14 & 0.75 & $0.51,1.08$ & 0.12 \\
\hline
\end{tabular}

*Reference is patients with classical mood stabilizer; †Dispensing of an anti-diabetic or a lipid-lowering drug; ‡Hazard ratio adjusted for age and gender; §95\% confidence interval;

ПSecond generation antipsychotic; **First generation antipsychotic. 
with an incident dispensing of lipid-lowering drugs were significantly less often exposed to SGAPs. These findings were not modified after excluding patients exposed to sedative FGAPs over the 3 month inclusion period (Table 2) or after adjusting for non-psychotropic drugs likely to induce metabolic events (results not shown).

\section{Second method: cumulative duration of exposure to antipsychotic drugs}

When exposure to antipsychotics was defined as cumulative exposure duration in number of days, i.e. person-days (Table 3), exposure to FGAPs or SGAPs was associated neither with the occurrence of an incident metabolic event nor with the dispensing of a new anti-diabetic or a new lipid-lowering drug. In analyses performed after excluding patients exposed to sedative FGAPs over the 3 month inclusion period, exposure to FGAPs was significantly associated with incident lipid-lowering drug dispensing, with an $\mathrm{HR}$ very close to 1 (Table 3).

After adjustment for diuretic or thyroid hormone use, cumulative exposure to SGAPs was significantly associated with an incident dispensing of a lipid-lowering drug ( $\mathrm{HR}$ $1.002,95 \% \mathrm{Cl} 1.000,1.003, P=0.045)$. Adjustment for the other non-psychotropic drugs likely to induce metabolic events did not modify the associations between metabolic events and cumulative duration of exposure to FGAPs or SGAPs, compared with conventional mood stabilizers (data not shown).

\section{Third method: exposure to antipsychotic drugs categorized in current and recent use}

In this case, the occurrence of an incident metabolic event, i.e. dispensing of an anti-diabetic or a lipidlowering drug, was associated with recent exposure to SGAPs, compared with conventional mood stabilizers (Table 4) but not with current exposure to SGAPs or with current or recent exposure to FGAPs. There was no significant association between anti-diabetic dispensing and exposure to antipsychotics. A new dispensing of a lipidlowering drug was associated with recent exposure to SGAPs or FGAPs, compared with exposure to conventional mood stabilizers. Analyses performed excluding patients exposed to sedative FGAPs over the 3 month inclusion period showed similar findings (Table 4). Adjustment for non-psychotropic drugs likely to induce metabolic events did not modify the associations between

\section{Table 3}

Association between cumulative exposure to antipsychotic drug treatment* and occurrence of incident metabolic event +

\begin{tabular}{|c|c|c|c|c|c|c|c|c|c|}
\hline \multirow[b]{2}{*}{ In total sample } & \multicolumn{3}{|c|}{ Incident anti-diabetic dispensing } & \multicolumn{3}{|c|}{ Incident lipid-lowering drug dispensing } & \multicolumn{3}{|c|}{ Any incident metabolic event ${ }^{\dagger}$} \\
\hline & HR $¥$ & $95 \%$ CIS & $P$ & HR $\ddagger$ & $95 \%$ CI§ & $P$ & HR‡ & $95 \%$ CIS & $P$ \\
\hline Cumulative exposure to SGAPף $t \dagger$ & 0.997 & $0.987,1.006$ & 0.49 & 1.001 & $0.999,1.003$ & 0.08 & 1.001 & $0.999,1.003$ & 0.20 \\
\hline \multicolumn{10}{|c|}{ In population excluding patients exposed to sedative antipsychotics over 3-month inclusion period } \\
\hline Cumulative exposure to SGAPף|t & 0.998 & $0.991,1.006$ & 0.75 & 1.001 & $0.999,1.003$ & 0.22 & 1.001 & $0.999,1.003$ & 0.29 \\
\hline Cumulative exposure to FGAP**t† & 0.987 & $0.959,1.015$ & 0.36 & 1.002 & $1.001,1.004$ & 0.006 & 1.000 & $0.997,1.003$ & 0.86 \\
\hline
\end{tabular}

* Reference is patients with classical mood stabilizer; †Dispensing of an anti-diabetic or a lipid-lowering drug; \#Hazard ratio adjusted for age and gender; §95\% confidence interval; ПSecond generation antipsychotic; **First generation antipsychotic; †+unit used in these analyses is number of days with drug exposure.

\section{Table 4}

Association between current* or recent† antipsychotic drug treatment and occurrence of incident metabolic eventł

\begin{tabular}{|c|c|c|c|c|c|c|c|c|c|}
\hline \multirow[b]{2}{*}{ In total sample } & \multicolumn{3}{|c|}{$\begin{array}{l}\text { Incident anti-diabetic } \\
\text { dispensing }(n=2990) \uparrow\end{array}$} & \multicolumn{3}{|c|}{$\begin{array}{l}\text { Incident lipid-lowering drug } \\
\text { dispensing }(n=2958) \uparrow\end{array}$} & \multicolumn{3}{|c|}{$\begin{array}{l}\text { Any incident metabolic } \\
\text { event } \S(n=2973) \uparrow\end{array}$} \\
\hline & $\mathrm{HR}^{* *}$ & $95 \% \mathrm{Clt}+$ & $P$ & $\mathrm{HR}^{* *}$ & $95 \% \mathrm{Cltt}$ & $P$ & $\mathrm{HR}^{* *}$ & $95 \% \mathrm{Cltt}$ & $P$ \\
\hline Current* treatment with SGAP $\neq \neq$ & 0.6 & $0.1,4.0$ & 0.6 & 0.8 & $0.4,1.7$ & 0.6 & 0.7 & $0.3,1.4$ & 0.3 \\
\hline Recentt treatment with SGAP¥¥ & 1.8 & $0.4,7.5$ & 0.4 & 2.3 & $1.3,4.0$ & 0.005 & 2.1 & $1.2,3.7$ & 0.006 \\
\hline Current* treatment with FGAP§§ & 1.2 & $0.5,2.7$ & 0.7 & 1.0 & $0.7,1.5$ & 0.9 & 0.9 & $0.6,1.3$ & 0.6 \\
\hline Recentt treatment with FGAP§§ & 0.7 & $0.2,3.1$ & 0.7 & 1.7 & $1.1,2.6$ & 0.03 & 1.4 & $0.9,2.2$ & 0.1 \\
\hline \multicolumn{10}{|c|}{ In population excluding patients exposed to sedative antipsychotics over 3 month inclusion period } \\
\hline Current* treatment with SGAP $\neq$ & 0.7 & $0.1,5.4$ & 0.8 & 0.7 & $0.3,1.7$ & 0.4 & 0.6 & $0.2,1.5$ & 0.3 \\
\hline Recentt treatment with SGAP¥¥ & 2.2 & $0.5,9.1$ & 0.3 & 2.2 & $1.2,4.1$ & 0.01 & 2.2 & $1.2,3.9$ & 0.01 \\
\hline Current* treatment with FGAP§§ & 0.6 & $0.1,4.1$ & 0.6 & 1.0 & $0.5,1.9$ & 0.9 & 0.8 & $0.4,1.7$ & 0.6 \\
\hline Recentt treatment with FGAP§§ & 0.6 & $0.1,4.5$ & 0.6 & 1.9 & $1.1,3.4$ & 0.03 & 1.4 & $0.8,2.4$ & 0.3 \\
\hline
\end{tabular}

*At time of metabolic event; +Interrupted for less than 6 months at time of metabolic event; ¥Reference is patients with classical mood stabilizer; §Dispensing of an anti-diabetic or a lipid-lowering drug; ๆNumber of patients with a current or recent dispensing of antipsychotic drug or conventional mood stabilizer at time of metabolic event; ** Hazard ratio adjusted for age and gender; ††95\% confidence interval; ‡¥Second generation antipsychotic; §§First generation antipsychotic. 
metabolic events and current or recent exposure to FGAPs or SGAPs (data not shown).

\section{Discussion}

A striking finding was the contrasting results ensuing from the three different definitions of antipsychotic exposure used in the present study. Patients with an incident metabolic event were less often exposed to any drugs likely to induce metabolic events over the study period than those without metabolic events. When considering the cumulative duration of exposure, FGAPs or SGAPs were not found associated with the occurrence of metabolic events. The occurrence of any metabolic event was significantly associated with recent exposure to SGAPs but not with current exposure to SGAPs or recent or current exposure to FGAPs, compared with exposure to conventional mood stabilizers. The occurrence of a lipid-lowering drug dispensing was associated with recent exposure to SGAPs or FGAPs, but not with current exposure.

The crucial importance of a precise and relevant timewindow definition of drug exposure is underlined by these findings. Drug exposure is a time-varying determinant and episodes of no use, past use and current use should be distinguished over the study period to avoid any nondifferential misclassification bias that might lead to negative findings [24, 25]. Indeed considering at least one dispensing of an antipsychotic over the study period led to considering subjects as exposed at the time of the event occurrence, whereas the event might have happened before the drug exposure or a long time after its discontinuation.

A plausible explanation of the association between the metabolic event and recent but not current exposure to antipsychotic drug treatment is that antipsychotic drug treatment led to the occurrence of a metabolic disturbance and then was discontinued because of this adverse effect and not prescribed again. This association may be explained by the delay between the occurrence of the metabolic disturbance (biological changes and/or weight gain), leading to treatment discontinuation and nutritional interventions for a few months, and the introduction of a lipid-lowering or an anti-diabetic drug treatment if lifestyle interventions did not prove adequate. A second explanation is that subjects prone to metabolic disturbances may discontinue antipsychotic drug treatments after a short period of treatment because of the occurrence of a metabolic change. As these persons are no longer in the category of current users of antipsychotics, this tends to decrease the strength of the association between current exposure to antipsychotics and incident metabolic events, corresponding to the so-called depletion of susceptibles effect [26]. Thus, patients with prevalent use of drugs are less likely to present with adverse effects of these drugs than those with incident use (i.e. who are experiencing these drugs for the first time), which may explain why patients who discontinued the treatment were more likely to have presented adverse events with these drugs and why no association was found between metabolic events and current use of antipsychotics or cumulative duration of exposure. Moreover, the impact of the cumulative duration of drug exposure might be unidentified, as subjects with metabolic changes might receive shorter periods of antipsychotic treatment than subjects without. Some studies supported the time-dependent exposure definition to assess the effectiveness of drugs such as statins [27], or the tolerance of drugs such as COX-2 inhibitors [28]. Nevertheless, the impact of these drugs might require a longer duration of exposure than for the metabolic effect of antipsychotics that may have occurred from the beginning of the treatment $[29,30]$.

The discrepant findings obtained using different measures of drug exposure in the same sample were individually concordant with those reported by studies funded by drug companies using a single measure in a given sample. Thus, some studies investigating the product marketed by the sponsor have considered the duration of antipsychotic exposure and found no difference or a weak one between FGAPs and SGAPs [5-7]. On the other hand, other studies investigating competing products of the sponsor considered the exposure to antipsychotic as a dichotomous variable and showed that quetiapine, risperidone [8, 9], olanzapine [8-10,31] and clozapine [8] were associated with an increased risk of diabetes relative to FGAPs.

The present study has some methodological limitations. The first concerns the selection of our study population with respect to the target population. Patients with bipolar disorders were identified through the use of conventional mood stabilizers only approved for bipolar disorder (lithium, valpromide and sodium divalproate). Consequently, patients presenting with diagnoses such as personality disorders or impulse control disorders may have been included in the study sample, as anticonvulsants may be used in clinical practice to treat such conditions. However, our inclusion criteria were probably more under-inclusive than over-inclusive, as many bipolar patients receive other conventional mood stabilizers or other psychotropic drugs, such as antipsychotics. Moreover, if this methodology induced the inclusion of subjects prescribed lithium, valpromide or sodium divaproate for another indication, this was not likely to invalidate the results of the paper. A second limitation concerns the assessment of outcome. The identification of an incident metabolic event would have implied appropriate biological monitoring of the patient and drug treatment. Patients with a metabolic event who were not routinely monitored or treated only using lifestyle and nutritional interventions [20] could have been misclassified as non-cases. Consequently, non-cases tended to be more like cases, which may bias risk estimates toward the null hypothesis. Conversely, patients treated with antipsychotic treatment may 
be more frequently monitored for metabolic disturbances. Therefore metabolic events could be more likely diagnosed in such patients. However, because screening for metabolic disturbance is often insufficient in clinical practice $[32,33]$, it is sensible to consider that patients treated or not by antipsychotics did not differ in terms of screening. Another limitation is that our sample may not be representative of the general population, as only selfemployed persons being insured by the RSI health care programme were included. However, these persons have the same rights regarding refunding of drugs and choice of treating physicians as those insured by the other health care programmes in France. As in any observational study, subjects were not randomized in treatment groups. Thus, patients with mood stabilizer treatment, FGAPs or SGAPs may differ in lifestyle and metabolic risk. Furthermore, we might lack statistical power owing to the small number of exposed cases to find any association between antipsychotic drug treatments and diabetes, compared with mood stabilizers. The diabetogenic effect of drugs might also require a longer length of exposure and thus a longer follow-up period [34]. Finally, administrative drug databases include drugs actually dispensed and do not indicate whether the drugs were taken as prescribed, which may lead to an overestimation of any drug exposure. This limitation is inherent to any observational study.

In conclusion, compared with other studies, two major strengths of the present study with respect to the assessment of exposure to antipsychotics were to consider only incident use of antipsychotic drugs and to investigate exposure to antipsychotic drugs in the 6 month period prior to the occurrence of metabolic events. Such findings would not have been reported if we had only considered current use of antipsychotic drugs using a cross-sectional design or the cumulative duration of exposure or if we had not defined a relevant time-window of exposure. Thus, the definition of exposure to antipsychotics in epidemiological studies exploring their metabolic impact is of paramount importance in understanding this association. Different definitions can lead to opposite and seemingly nonsensical results. Results from research in this field have to be approached very cautiously and firm differential conclusions may not be possible. Nevertheless people on any antipsychotic or mood stabilizer should be regularly assessed for metabolic changes.

\section{Competing Interests}

There are no competing interests to declare.

We thank Ray Cooke for supervising the English of this manuscript. This was an independent study funded by autonomous resources of INSERM U657.

\section{REFERENCES}

1 Geddes J, Freemantle N, Harrison P, Bebbington P. Atypical antipsychotics in the treatment of schizophrenia: systematic overview and meta-regression analysis. BMJ 2000; 321: 1371-6.

2 Balf G, Stewart TD, Whitehead R, Baker RA. Metabolic adverse events in patients with mental illness treated with antipsychotics: a primary care perspective. Prim Care Companion J Clin Psychiatry 2008; 10: 15-24.

3 Meyer JM, Stahl SM. The metabolic syndrome and schizophrenia. Acta Psychiatr Scand 2009; 119: 4-14.

4 Stahl SM, Mignon L, Meyer JM. Which comes first: atypical antipsychotic treatment or cardiometabolic risk? Acta Psychiatr Scand 2009; 119: 171-9.

5 Buse JB, Cavazzoni P, Hornbuckle K, Hutchins D, Breier A, Jovanovic L. A retrospective cohort study of diabetes mellitus and antipsychotic treatment in the United States. J Clin Epidemiol 2003; 56: 164-70.

6 Ostbye T, Curtis LH, Masselink LE, Hutchison S, Wright A, Dans PE, Schulman KA, Krishnan RR. Atypical antipsychotic drugs and diabetes mellitus in a large outpatient population: a retrospective cohort study. Pharmacoepidemiol Drug Saf 2005; 14:407-15.

7 Carlson C, Hornbuckle K, DeLisle F, Kryzhanovskaya L, Breier A, Cavazzoni P. Diabetes mellitus and antipsychotic treatment in the United Kingdom. Eur Neuropsychopharmacol 2006; 16: 366-75.

8 Guo JJ, Keck PE Jr, Corey-Lisle PK, Li H, Jiang D, Jang R, L'Italien GJ. Risk of diabetes mellitus associated with atypical antipsychotic use among patients with bipolar disorder: a retrospective, population-based, case-control study. J Clin Psychiatry 2006; 67: 1055-61.

9 Guo JJ, Keck PE Jr, Corey-Lisle PK, Li H, Jiang D, Jang R, L'Italien GJ. Risk of diabetes mellitus associated with atypical antipsychotic use among Medicaid patients with bipolar disorder: a nested case-control study. Pharmacotherapy 2007; 27: 27-35.

10 Koro CE, Fedder DO, L'Italien GJ, Weiss SS, Magder LS, Kreyenbuhl J, Revicki DA, Buchanan RW. Assessment of independent effect of olanzapine and risperidone on risk of diabetes among patients with schizophrenia: population based nested case-control study. BMJ 2002; 325: 243.

11 Yumru M, Savas HA, Kurt E, Kaya MC, Selek S, Savas E, Oral ET, Atagun I. Atypical antipsychotics related metabolic syndrome in bipolar patients. J Affect Disord 2007; 98: 247-52.

12 Fountoulakis KN, Vieta E. Treatment of bipolar disorder: a systematic review of available data and clinical perspectives. Int J Neuropsychopharmacol 2008; 11:999-1029.

13 Verdoux $H$, Tournier M, Bégaud B. Antipsychotic prescribing trends: a review of pharmaco-epidemiological studies. Acta Psychiatr Scand 2010; 121:4-10.

14 Moller HJ. Antipsychotic and antidepressive effects of second generation antipsychotics: two different pharmacological mechanisms? Eur Arch Psychiatry Clin Neurosci 2005; 255: 190-201. 
15 Tohen M, Chengappa KN, Suppes T, Zarate CA Jr, Calabrese JR, Bowden CL, Sachs GS, Kupfer DJ, Baker RW, Risser RC, Keeter EL, Feldman PD, Tollefson GD, Breier A. Efficacy of olanzapine in combination with valproate or lithium in the treatment of mania in patients partially nonresponsive to valproate or lithium monotherapy. Arch Gen Psychiatry 2002; 59: 62-9.

16 Verdoux H, Cougnard A, Auleley GR, Deligne J, Blum-Boisgard C, Bégaud B, Tournier M. Antipsychotic use patterns in persons initially treated with mood stabilizers: a naturalistic study. Pharmacopsychiatry 2010; 43: 17-23.

17 WHO Collaborating Centre for Drug Statistics Methodology. About the ATC/DDD System. Oslo: Norwegian Institute of Public Health, 2007.

18 Chan JC, Cockram CS, Critchley JA. Drug-induced disorders of glucose metabolism. Mechanisms and management. Drug Saf 1996; 15: 135-57.

19 Bantle JP, Wylie-Rosett J, Albright AL, Apovian CM, Clark NG, Franz MJ, Hoogwerf BJ, Lichtenstein AH, Mayer-Davis E, Mooradian AD, Wheeler ML. Nutrition recommendations and interventions for diabetes - 2006: a position statement of the American Diabetes Association. Diabetes Care 2006; 29: 2140-57.

20 Morrison F, Shubina M, Turchin A. Lifestyle counseling in routine care and long-term glucose, blood pressure, and cholesterol control in patients with diabetes. Diabetes Care 2012; 35: 334-41.

21 Cox DR. Regression models and life tables. J R Stat Soc 1972; B34: 187-220.

22 Hertz-Picciotto I, Rockhill B. Validity and efficiency of approximation methods for tied survival times in Cox regression. Biometrics 1997; 53: 1151-6.

23 StataCorp. Stata Statistical Software: Release 9. College Station, TX: StataCorp LP, 2005.

24 Stricker BH, Stijnen T. Analysis of individual drug use as a time-varying determinant of exposure in prospective population-based cohort studies. Eur J Epidemiol 2010; 25: 245-51.
25 Suissa S. Immortal time bias in pharmaco-epidemiology. Am J Epidemiol 2008; 167: 492-9.

26 Moride Y, Abenhaim L. Evidence of the depletion of susceptibles effect in non-experimental pharmacoepidemiologic research. J Clin Epidemiol 1994; 47: 731-7.

27 Maitland-van der Zee $\mathrm{AH}$, Klungel $\mathrm{OH}$, Stricker $\mathrm{BH}$, Verschuren WM, Witteman JC, Stijnen T, Hofman A, Breteler MM, Leufkens HG, de Boer A. Comparison of two methodologies to analyze exposure to statins in an observational study on effectiveness. J Clin Epidemiol 2004; 57: 237-42.

28 Motsko SP, Rascati KL, Busti AJ, Wilson JP, Barner JC, Lawson KA, Worchel J. Temporal relationship between use of NSAIDs, including selective COX-2 inhibitors, and cardiovascular risk. Drug Saf 2006; 29: 621-32.

29 Allison DB, Mentore JL, Heo M, Chandler LP, Cappelleri JC, Infante MC, Weiden PJ. Antipsychotic-induced weight gain: a comprehensive research synthesis. Am J Psychiatry 1999; 156: 1686-96.

30 Meyer JM, Davis VG, Goff DC, McEvoy JP, Nasrallah HA, Davis SM, Rosenheck RA, Daumit GL, Hsiao J, Swartz MS, Stroup TS, Lieberman JA. Change in metabolic syndrome parameters with antipsychotic treatment in the CATIE Schizophrenia Trial: prospective data from phase 1. Schizophr Res 2008; 101: 273-86.

31 Koro CE, Fedder DO, L'Italien GJ, Weiss S, Magder LS, Kreyenbuhl J, Revicki D, Buchanan RW. An assessment of the independent effects of olanzapine and risperidone exposure on the risk of hyperlipidemia in schizophrenic patients. Arch Gen Psychiatry 2002; 59: 1021-6.

32 Jennex A, Gardner DM. Monitoring and management of metabolic risk factors in outpatients taking antipsychotic drugs: a controlled study. Can J Psychiatry 2008; 53: 34-42.

33 Verdoux H, Boulon S, Cougnard A. Gender differences in metabolic monitoring of second-generation antipsychotic prescription. Hum Psychopharmacol 2008; 23: 471-4.

34 Bushe CJ, Leonard BE. Blood glucose and schizophrenia: a systematic review of prospective randomized clinical trials. J Clin Psychiatry 2007; 68: 1682-90. 